\title{
COVID-19: Prolonged Social Distancing Implementation Strategy Using Blockchain-Based Movement Passes
}

\author{
Chandan Garg $^{1} \cdot$ Agam Bansal $^{2} \cdot$ Rana Prathap Padappayil ${ }^{3}$
}

Received: 17 July 2020 / Accepted: 21 July 2020 / Published online: 11 August 2020

(C) Springer Science+Business Media, LLC, part of Springer Nature 2020

With about dozen countries officially remaining COVID-19 free as of writing this article, most of the world has laid down some form of social distancing guidelines to combat the spread of the virus. Currently, with the lack of vaccine and effective medical therapies being in various stages of clinical trials, social distancing is the only measure to help curb the spread of the infection [1]. The most effective method of implementing social distancing is by reducing the number of persons in any given place at a given time. State and national authorities have attempted to promote this by laying down regulations on the number of people who could attend an indoor or outdoor event at any given point of time. Since premature relaxation of regulations would undo the benefits rendered by previous social distancing efforts by making the society vulnerable to a second wave of the infection, we need to explore strategies of sustainable social distancing and crowd reduction strategies that can be easily implemented over prolonged periods of time.

Many European nations have opted to reopen businesses in a phased pattern, with smaller shops and businesses opening early followed by schools, and restaurants, cafes, and cinemas, etc. opening much later. Countries such as India have opted to categorize the entire nation into zones based on COVID-19 case density and reopen businesses in the lower risk zones first. Another proposed strategy is to allow partial lifting of the lockdown restrictions during certain pre-designated times of the day so that businesses can function at a limited capacity

This article is part of the Topical Collection on Patient Facing Systems

Chandan Garg

olf.chandan@gmail.com

1 Department of Statistics, Columbia University, New York, NY, USA

2 Department of Internal Medicine, Cleveland Clinic, Cleveland, $\mathrm{OH}$, USA

3 Department of Emergency Medicine, Brigham and Women's Hospital, Boston, MA, USA only during those times. However, these strategies raise the concern that due to the prolonged nature of the currently active lockdown, the public might be tempted to go out in large numbers once the restrictions ease in, and that would lead to an increased person to person contact and theoretically higher chances of spread of the infection. Besides, it becomes imperative that as long as there are no vaccines available, return to pre-lockdown normal may not be possible in many places, and there would be a continued focus on social distancing and crowd-reduction measures. There is a real need for a robust social distancing implementation strategy that authorities can follow and continue until effective tools to prevent the spread of the disease become available [2].

We propose a blockchain-based system to allow authorities to promote social distancing by allowing only a specific number of individuals at any given time in a designated area. Blockchain technology enables us to conduct electronic transactions or transfer of privileged information among participants of a network without the need of a centralized trusted third party ledger [3]. Started initially as a decentralized financial tool, the current iteration of blockchain technology (Blockchain 3.0) aims to expand the utilities of this technology beyond the financial market, and well into healthcare industries. "Smart Contracts" is one of the aspects of this technology that allows securely storing records and information on a system-wide blockchain comprising of participants or nodes [4]. Storing information on a decentralized ledger such as a blockchain network instead of on a centralized ledger such as a hospital network serves several advantages, the first of which is participant privacy. When a participant becomes part of the blockchain, a pair of keys will be generated. The private key resides with the participant whereas the public key resides on the chain. Using smart contracts, privileged information is released to the private key holder only when their private key matches the public key. By linking the private key to a person's biometrics, this access system becomes fast, secure, and versatile.

Citizens can voluntarily sign up to a blockchain-based mobile application (blockchain wallet) which would 
provide them with "movement passes" or time-based movement tokens which can be spent by the person multiple times until it expires. Police, city mayors, or government departments can easily verify these passes to ensure that only those with an active movement pass is in a public space and conducting business at any given time. By restricting the total number of movement passes that are active during a certain time of the day, the crowd in public places can be controlled. This solution can be easily scaled up, from smaller companies attempting to limit the number of workers in a given day to larger populations and counties limiting the number of persons attending an event. The use of a mobile application ensures that this system is adaptable to a variety of situations, such as restricting entries into grocery stores to only those with a movement pass, establishing roadside checkpoints, etc. Additionally, the interoperability of blockchain with the existing banking network can be tapped in, to explore the option of limiting financial transactions to only those with an active movement pass, to de-incentivize violations.

The government would jointly run the blockchain (nodes with weighted votes and this could be the federal government, state government, city mayors, or the police department) with the citizens. The citizens can sign up to their mobile wallets using biometrics as a private key, and while doing so, a public key would be generated. Government database has biometric data linked to citizens' profiles like geography, family size, vehicle details, income level, international travel history, etc. Smart Contracts can be used to link this profile data with their respective blockchain wallet, thereby maintaining the anonymity and privacy of the citizens.

This profile data can be used to develop a staggered exit strategy allowing people from a specific cluster, demographics, geographic location, or occupation, to move out at a particular time to avoid crowding at public places. Once the time slot for the citizens is assigned, this gets tagged to their wallet to allow verification in their respective time slots. For verification purposes, the person is required to do a transaction sending a movement token to the verifying officer. If the pass is valid and the person is the actual owner of the token, the nodes will update the transaction in the ledger and the verification is complete. In case of invalid token or fraudulent login, the nodes won't be able to update the ledger and the person fails to verify (Fig. 1).

Our proposed solutions accept and interpret data from multiple governmental and hospital networks and can inturn be programmed to identify high risked individuals, case tracking, contact tracing, early isolation for testing, and quarantining. In addition to the government profile data, other data points such as those from digital phenotyping (geolocation, travel history or proximity with other known COVID-19 patients), medical records which may be linked to the government profile data, can also be added to the data pool and large-scale machine learning may be used to aid the time slot allocation. In addition to the government assigned movement pass, there can also be government-issued "immunity certificates" which act complementary to each other through blockchain. Immunity certificates can be issued by the government anonymously based on data from medical records of previous COVID-19 infection or vaccination status when a vaccine becomes available.

Using blockchain for such an attempt provides several advantages. Linkage to biometrics ensures that one's movement pass cannot be used by anyone else. This also provides immense flexibility and scalability at the level of the

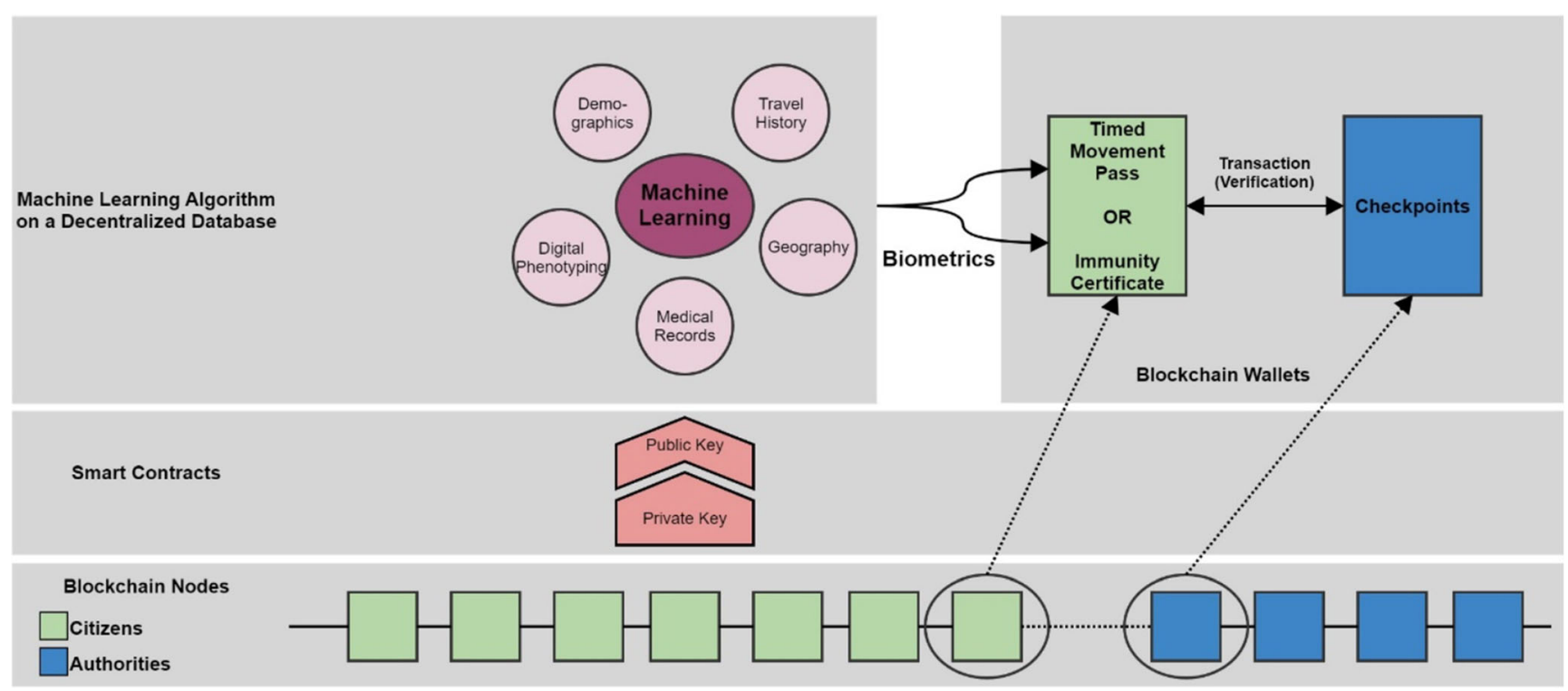

Fig. 1 An overview of how blockchain technology can validate a movement pass or immunity certificate generated by a machine learning algorithm 
government. For example, if a localized new outbreak is suspected, then the movement passes in that area can be limited until case identification and contact tracing is complete. Blockchain is tamper-proof and neither the government nor the citizens can replicate or reuse the movement passes that are issued by the system to individuals. Even though the system relies on large scale data processing, there is complete privacy of the individual and hence acceptability could be very good in the right setting.

There can be large-scale implementation hurdles to such a system. One of the major drawbacks of the system is that it relies on participants owning and operating a smartphone. Despite the heavy penetration of smartphones in recent years, participant willingness to always carry a smartphone to move around may not be acceptable in some populations. In addition, the ethical and humanitarian concerns to such an implementation will need to be examined prior to a large-scale roll out. Small pilot trials can help and identify participant concerns in using the technology. However, several smartphone-based tracking and digital phenotyping efforts were largely the reason behind Taiwan's success in reducing COVID-19 case numbers, and blockchain-based movement passes could be a valuable extension to such an effort in reducing crowds in public places and promoting social distancing [5].

\section{Compliance with ethical standards}

Conflict of interest The authors declare no conflicts of interest.

Ethical approval This article does not contain any studies with human participants or animals performed by any of the authors.

\section{References}

1. Bonell C, Michie S, Reicher S, West R, Bear L, Yardley L, et al. Harnessing behavioural science in public health campaigns to maintain "social distancing" in response to the COVID-19 pandemic: key principles. J. Epidemiol. Community Health 2020.

2. Petersen E, Wasserman S, Lee S-S, Go U, Holmes AH, Abri SA, et al. COVID-19-We urgently need to start developing an exit strategy. Int. J. Infect. Dis. IJID Off. Publ. Int. Soc. Infect. Dis. 2020.

3. Agbo CC, Mahmoud QH, Eklund JM. Blockchain technology in healthcare: A systematic review. Healthcare [Internet]. 2019 Apr 4 [cited 2020 May 10];7(2). Available from: https://www.ncbi.nlm. nih.gov/pmc/articles/PMC6627742/.

4. Giordanengo A. Possible usages of smart contracts (blockchain) in healthcare and why no one is using them. Stud Health Technol Inform. 2019;264:596-600.

5. Wang CJ, Ng CY, Brook RH. Response to COVID-19 in Taiwan: Big data analytics, new technology, and proactive testing. JAMA. 2020;323(14):1341-2.

Publisher's note Springer Nature remains neutral with regard to jurisdictional claims in published maps and institutional affiliations. 